\title{
Компьютерное моделирование структуры технических поверхностей на микроуровне
}

\author{
A.A. Рачишкин 1, к.т.н., доиент, RachishkinAndr@yandex.ru \\ O.B. Сутягин 1, д.m.н., доиент, sutyagine@rambler.ru \\ A.H. Болотов 1, д.m.н., npodeccop, alnikbltov@rambler.ru
}

\author{
1 Тверской государственный технический университет, г. Тверь, 170026, Россия
}

В статье описывается структура программного средства для компьютерного моделирования топографии технических поверхностей. Модель построена с использованием экспериментальных данных о морфологии шероховатых поверхностей. Это позволяет моделировать трехмерную поверхность, используя данные о характеристиках микротопографии контактирующих поверхностей. При неполных данных или их отсутствии можно использовать статистически обработанные параметры продольных и поперечных профилей поверхностей.

С учетом возможной неоднородности объекта исследований генерация микронеровностей осуществляется по специально разработанному математическому аппарату. Принятые допущения не оказывают существенного влияния на достоверность моделируемой поверхности. Такой подход позволяет проектировать неоднородные шероховатые поверхности, исходя из индивидуальных параметров каждой микронеровности.

Проведенные аналитические расчеты и экспериментальные проверки показали сходимость в пределах допустимой погрешности моделируемой и реальной поверхностей. Расхождения обусловлены неоднородностью реальной шероховатой поверхности. Полное копирование топографии технически реализуемо, но ведет к невозможности построения расчетных моделей для исследования физических процессов, протекающих при контактном взаимодействии двух поверхностей. Таким образом, подход с принятием допущений представляется оптимальным решением при проектировании компьютерной модели, являющейся базовым модулем программы для исследования процесса взаимодействия шероховатых поверхностей с возможностью нанесения функционального покрытия.

Разработанный математический аппарат и принятые входные параметры позволяют имитировать реальную шероховатую поверхность с максимально возможной достоверностью. Могут осуществляться как моделирование существующих шероховатых поверхностей с известными значениями входных параметров по обработке, так и предварительное проектирование наиболее оптимальной топографии поверхности, необходимой для решения конкретной инженерной задачи. Данная программа объединяет современные информационные технологии и аналитические разработки в области генерации шероховатых поверхностей. Она является отдельным, базовым модулем общей системы моделирования узлов трибосопряжений.

Ключевые слова: компьютерное моделирование, шероховатая поверхность, трехмерная модель, микронеровность, топография поверхности.

Разработка и создание компьютерных моделей для исследования микротопографии и контактного взаимодействия шероховатых поверхностей является актуальной задачей современной инженерной практики.

Для построения трехмерной компьютерной модели шероховатой поверхности в работе [1] использовался математический аппарат, основанный на быстром преобразовании Фурье. В такой модели определение области фактического контакта происходило на основании геометрической интерференции двух контактирующих моделей шероховатых поверхностей. Далее математический аппарат модели построен так, что расчет площади фактического контакта и контактных давлений происходил путем итерационного процесса с инверсией матрицы, содержащей дискретные ячейки областей фактического контакта. Увеличение числа пятен фактического контакта перегружало вычислительную систему, поэтому для ускорения вычислительного процесса авторам пришлось моделировать минимальный фрагмент взаимодействующих поверхностей и энергетический подход при анализе контактных деформаций микронеровностей $[1,2]$.
Это не позволяло адекватно оценивать напряженно-деформированное состояние на пятнах фактического контакта, что существенно снижало достоверность получаемых результатов.

Применение теории случайного поля позволяет получать спектральные характеристики поверхностей и использовать их для решения периодической задачи теории упругости с синусоидальным штампом. Такой подход может быть использован для моделирования контактных характеристик [3], однако ограничен только упругими деформациями микронеровностей.

Контактные задачи имеют решения только для тел с правильной геометрической формой, поэтому при создании моделей микронеровностей, образующих технические поверхности, используются клинья, стержни, сегменты цилиндров и сфер [4].

Разработка программного средства для компьютерного моделирования взаимодействий микронеровностей технических поверхностей должна была объединить существующие подходы к прогнозированию характеристик контакта шероховатых поверхностей и современные компьютерные технологии [5-7]. Синтез такой модели может быть 
основан на применении системного подхода. Для его реализации на первом этапе необходимо выделить в системе условно автономные элементы. Это позволяет в дальнейшем добавлять, изменять и отлаживать модули программы, не нарушая ее структуру. Применительно к рассматриваемой задаче это может выглядеть так, как представлено на обобщенной схеме (рис. 1).

Входные значения параметров запрашиваются последовательно на каждом этапе моделирования. Модули синтеза топографии поверхности и расчет характеристик контактного взаимодействия необходимы для выполнения каждого из дополнительных элементов, которые задействуются в зависимости от поставленной задачи.

Генерация топографии поверхности. Создание модели трехмерной шероховатой поверхности позволяет максимально приблизить результаты моделирования к данным, полученным экспериментальным путем [8]. При создании модели следует учитывать, что для большинства задач контактного взаимодействия нужно рассматривать только часть слоя, состоящую из выступов шероховатой поверхности [9]. Поэтому моделирование поверхности, расположенной ниже средней плоскости, опускается.

При создании математического аппарата принимается допущение, что каждая микронеровность шероховатой поверхности представляется как одинаково ориентированные сегменты эллипсоидов вращения. Для эффективного распределения их на плоскости создан алгоритм, учитывающий входные параметры и общую структуру поверхности. Высоты и радиусы неровностей изменяются по случайному закону распределения. Модель неровности в виде сегмента эллипсоида вращения учитывает анизотропию контактирующих поверхностей, а ее частным случаем является модель в виде

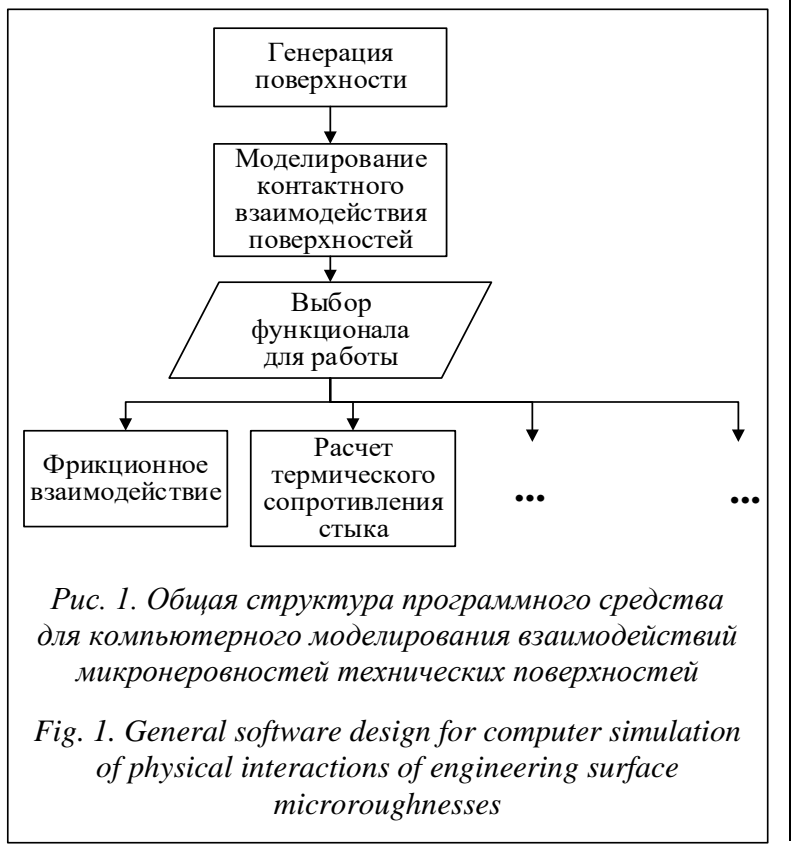

сферического сегмента, широко применяемая в исследованиях контактного взаимодействия [10]. Количества сгенерированных сегментов достаточно для статистического описания моделируемой шероховатой поверхности. Каждый сегмент эллипсоида вращения, моделирующий микронеровность, имеет собственные геометрические параметры и координаты центра основания, при этом основания соседних сегментов не пересекаются. Субмикрошероховатости поверхности не учитываются.

Техническими входными параметрами для моделирования микротопографии шероховатой поверхности служат следующие характеристики, описывающие каждую генерируемую модель микронеровности:

- высота;

- продольный и поперечный радиусы вершины;

- продольный и поперечный шаги;

- количество микронеровностей.

Эти характеристики задаются минимальным и максимальным значениями и генерируются для каждой неровности по закону бета-распределения.

Входными параметрами, вводимыми оператором для компьютерного моделирования структуры дискретного контакта на микроуровне, являются конкретные данные о характеристиках микротопографии контактирующих поверхностей, а при их отсутствии - статистически обработанные параметры продольных и поперечных профилей поверхностей по ГОСТ 25142-82:

- Rpx, Rpy - максимальная высота выступа шероховатой поверхности в продольном $(x)$ и поперечном (y) направлениях;

- Rax, Ray - среднее арифметическое отклонение профиля шероховатой поверхности в продольном $(x)$ и поперечном $(y)$ направлениях.

- $S m x, S m y$ - шаг неровностей по средней линии в продольном $(x)$ и поперечном $(y)$ направлениях;

- $t m x$, tmy - относительная опорная длина профиля по средней линии в продольном $(x)$ и поперечном $(y)$ направлениях.

На рисунке 2 отображена общая блок-схема алгоритма генерации поверхности. Вводимые оператором данные проверяются на допустимость, сокращая ошибки из-за человеческого фактора. Далее идет циклическая генерация каждой микронеровности, индивидуальные значения параметров самой микронеровности и координат ее размещения на поверхности проходят дополнительную проверку относительно соседних элементов рельефа. Это необходимо для исключения ситуаций, выходящих за рамки допущения модели. За размещение микронеровностей отвечает специальный алгоритм корректировки. Основными инструментами алгоритма являются перемещение координат расположения обрабатываемого объекта и регулирование генерируемых индивидуальных параметров объекта. 


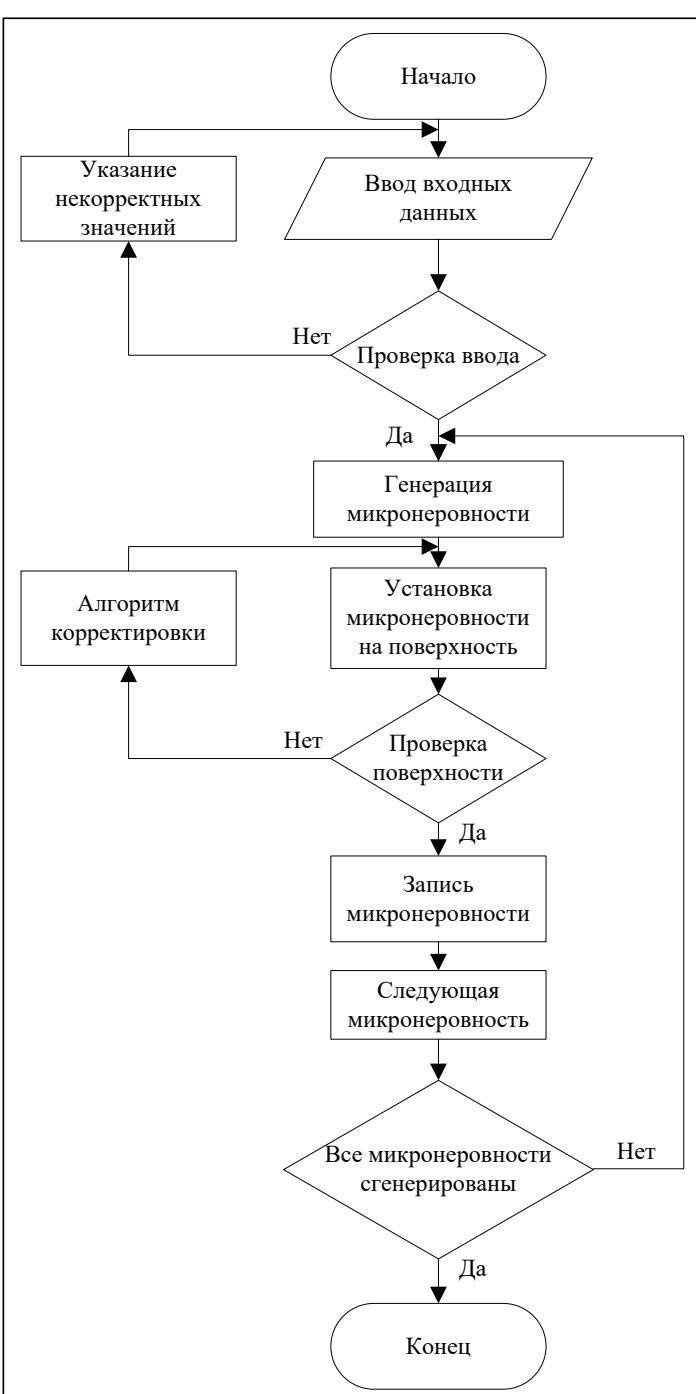

Рис. 2. Общая блок-схема генерации шероховатой поверхности

Fig. 2. A general block diagram of a rough surface generation

Данный алгоритм проявляет вмешательство в среднем в 30 \% случаев генерации микронеровности на поверхности, однако это не влияет на достоверность получаемых результатов, так как все корректировки проходят в допустимых значениях входных параметров.

Анализ сгенерированной поверхности. Поскольку топография поверхности - базовый этап моделирования, отвечающий за все последующие блоки, тестирование проводилось в несколько этапов. Первый этап - исследование простой детерминированной модели. Результаты обработки профилограмм в этом случае можно получить аналитически. Компьютерное моделирование данной ситуации показало полное совпадение с аналитическими результатами. Второй этап тестирования моделирование реальных поверхностей. Исследование параметров микрогеометрии реальных поверхностей проводилось на профилометре TR200
(Time Group Inc, China). В качестве объектов исследования использовались стальные образцы, поверхности которых обработаны торцевым фрезерованием. На рисунке 3 представлены модели шероховатых поверхностей. Проведено сопоставление со статистически обработанными графиками продольных и поперечных опорных кривых профилей моделей поверхностей и их реальных прототипов, где $t_{p}(\varepsilon)$ - относительная опорная длина профиля на относительном уровне $\varepsilon$. Для статистической обработки с моделей, как и с реальных поверхностей, снималось по пять продольных и поперечных профилограмм. На графиках треугольниками обозначены средние точки и доверительные интервалы опорных кривых профилей реальных поверхностей, прямоугольниками - средние точки и доверительные интервалы опорных кривых профилей моделей.

Статистически обработанные опорные кривые смоделированных профилограмм имеют удовле-
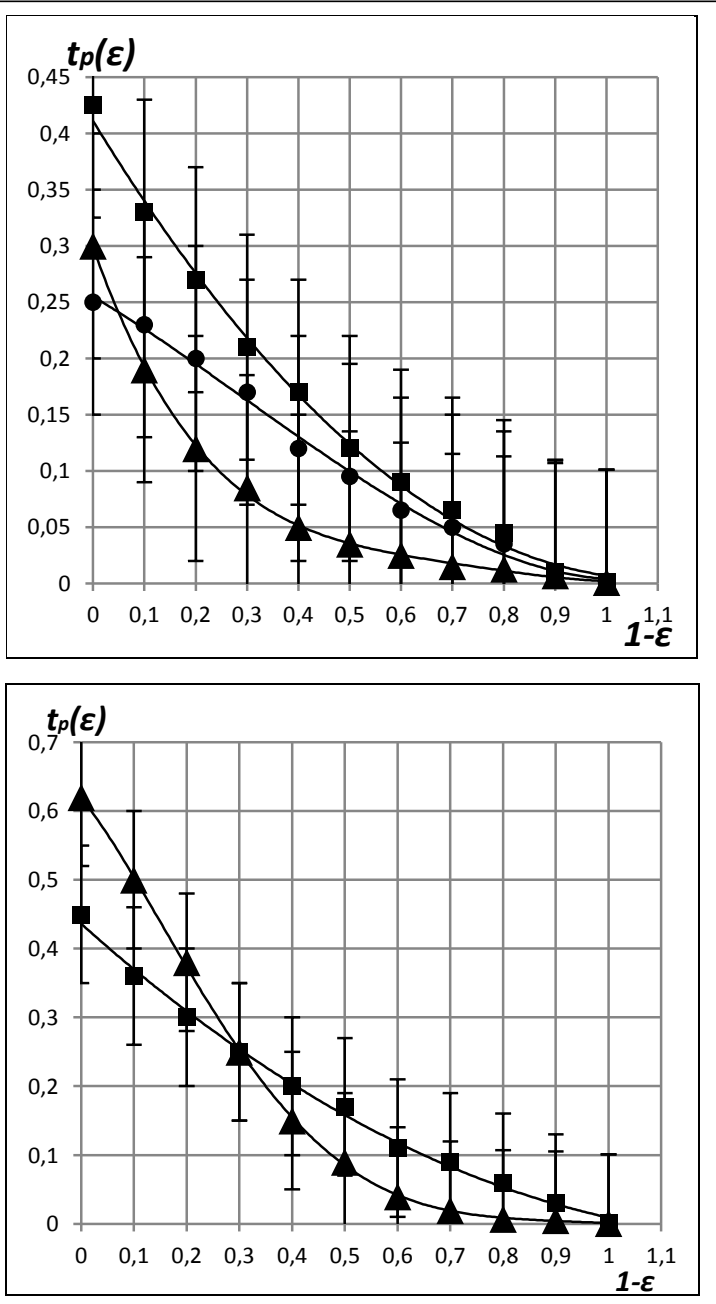

Рис. 3. Моделирование опорных кривых реальных шероховатых поверхностей по горизонтали и вертикали

Fig. 3. Horizontal and vertical simulation of supporting curves of real rough surfaces 
творительное соответствие со статистически обработанными опорными кривыми профилограмм реальных шероховатых поверхностей.

\section{Заключение}

Разработка подобной трехмерной компьютерной модели шероховатой поверхности является базой для построения множества сложных физических процессов, происходящих при контактном взаимодействии двух поверхностей. Связь современных информационных технологий и разработанных аналитических методик позволяет создать продукт, способный сократить временные и ресурсные затраты на проведение испытаний, проектирование методик обработки поверхностей и исследование наиболее подходящих функциональных покрытий.

\section{Литература}

1. Bhushan B., Peng W. Contact mechanics of multilayered rough surfaces. Appl. Mech. Rev. 2002, vol. 55, no. 5, pp. 435-480.
2. Bhushan B. Contact mechanics of rough surfaces in tribology: multiple asperity contact. Tribology letters, 1998, no. 4. pp. 1-35. DOI: 10.1023/A:1019186601445

3. Горячева И.Г., Чекина О.Г. Механика дискретного контакта. В кн.: Механика контактных взаимодействий; [под ред. И.И. Воровича и В.М. Александрова]. М.: Физматлит, 2001. С. $418-437$.

4. Крагельский И.В., Добычин М.Н., Комбалов В.С. Основы расчетов на трение и износ. М.: Машиностроение, 1977. $526 \mathrm{c}$.

5. Шилдт Г. Полное руководство С\# 4.0. М.: Вильямс, 2011. $1056 \mathrm{c}$.

6. Сутягин О.В., Болотов А.Н., Рачишкин А.А. Компьютерное моделирование контактного взаимодействия шероховатых поверхностей // Трение и износ. 2016. Т. 37. № 3. С. 267-268.

7. Демкин Н.Б., Измайлов В.В. Зависимость эксплуатационных свойств фрикционного контакта от микрогеометрии контактирующих поверхностей // Трение и износ. 2010. Т. 31. № 1. C. $68-77$.

8. Рачишкин А.А., Болотов А.Н., Сутягин О.В. Программа моделирования трехмерных шероховатых поверхностей. Свид. о гос. регистр. прогр. для ЭВМ № 2014661957; заявл. 29.07.2014; опубл. 20.12.2014.

9. Демкин Н.Б., Рыжов Э.В. Качество поверхности и контакт деталей машин. М.: Машиностроение, 1981. 224 с.

10. Горячева И.Г. Механика фрикционного взаимодействия. М.: Наука, 2001. 478 с.

\section{Computer simulation of engineering surface structure at the micro-level}

A.A. Rachishkin ${ }^{1}$, Ph.D. (Engineering), Associate Professor, RachishkinAndr@yandex.ru

O.V.Sutyagin ${ }^{1}$, Dr.Sc. (Engineering), Associate Professor, sutyagine@ rambler.ru

A.N. Bolotov ${ }^{1}$, Dr.Sc. (Engineering), Professor, alnikbltov@ rambler.ru

${ }^{1}$ Tver State Technical University, Tver, 170026, Russian Federation

Abstract This paper describes a software tool structure for computer simulation of an engineering surface topography. The model uses experimental data on the rough surface morphology. It is designed to model a 3D surface using characteristics data of contacting surface microtopography. In case of incomplete or absent data, we can use statistically processed parameters of longitudinal and transverse surface profiles.

Taking into account the possible inhomogenuity of the object of research, microroughness generation uses a specially developed mathematical apparatus. The assumptions made do not affect the simulated surface credibility considerably. This approach allows designing unhomogeneous rough surfaces based on individual parameters of each surface microroughness.

Analytical calculations and experimental tests showed convergence within the defined margin of error of the simulated and real surfaces. The actual rough surface inhomogeneity causes discrepancies. Full topography copying is technically feasible, but it leads to the impossibility of building computational models for studying the physical processes occurring during the contact interaction of two surfaces. Thus, making assumptions is the optimal solution when designing a computer model, which is the basic module of the program for studying the process of rough surface interaction with the possibility of applying functional coating.

The developed mathematical apparatus and the accepted input parameters make it possible to simulate a real rough surface with the highest possible reliability. It can be a simulation of existing rough surfaces with known processing parameters, or preliminary design of the most optimal surface topography that is necessary to solve a specific engineering task. This software combines modern information technologies and analytical developments of rough surface generation. It is a separate basic module of the friction unit modeling system.

Keywords: computer modeling, rough surface, 3D model, microroughness, surface topography.

\section{References}

1. Bhushan B., Peng W. Contact mechanics of multilayered rough surfaces. Appl. Mech. Rev. 2002, vol. 55, no. 5, pp. 435-480

2. Bhushan B. Contact mechanics of rough surfaces in tribology: multiple asperity contact. Tribology Letters. 1998, no. 4, pp. 1-35. DOI: $10.1023 / \mathrm{A}: 1019186601445$

3. Goryacheva I.G., Chekina O.G. Mechanics of contact interactions. Contact Mechanics. Moscow, Fizmatlit, 2001, pp. $418-437$ (in Russ.)

4. Kragelsky I.V., Dobychin M.N., Kombalov V.S. Basics of Friction and Wear Calculations. Moscow, Mashinostroyenie Publ., 1977, 516 p.

5. Schildt H. C\# 4.0 The Complete Reference. McGraw-Hill Education Publ., 2010, 976 p.

6. Sutyagin O.V., Bolotov A.N., Rachishkin A.A. Computer simulation of contact interaction of rough surfaces. J. of Friction and Wear. 2016, vol. 37, no. 3, pp. 267-268 (in Russ.).

7. Demkin N.B., Izmaylov V.V. The relation between the friction contact performance and the microgeometry of contacting surfaces. $J$. of Friction and Wear. 2010, vol. 31, no. 1, pp. 68-77 (in Russ.)

8. Rachishkin A.A., Bolotov A.N., Sutyagin O.V. A Program for Modeling 3-Dimensional Rough Surfaces. Certificate of computer program registration no. 2014661957, 2014.

9. Demkin N.B., Ryzhov E.V. Surface Quality and Contact of Machine Parts. Moscow, Mashinostroyenie, 1981, 224 p.

10. Goryacheva I.G. Frictional Interaction Mechanics. Moscow, Nauka Publ., 2001, 478 p. (in Russ). 